\title{
Les sepsis observés au service des maladies infectieuses du CHU Yalgado Ouédraogo de Ouagadougou : aspects épidémiologiques, cliniques et évolutifs.
}

\section{Sepsis observed in the infectious diseases department of the Yalgado Ouedraogo CHU (University Hospital) in Ouagadougou: clinical and evolutionary epidemiological aspects}

\author{
Savadogo $\mathrm{M}^{1}$, Diallo $\mathrm{I}^{2}$, Diendéré A E $\mathrm{E}^{3}$, Sondo $\mathrm{K} \mathrm{A}^{1}$, Sawadogo $\mathrm{A}^{4}$ \\ 1) Service des maladies infectieuses du CHU Yalgado Ouédraogo \\ 2) Service de médecine interne du CHU Yalgado Ouédraogo \\ 3) Service médecine du CHU de Bogodogo \\ 4) Service des maladies infectieuses, CHUR de Ouahigouya
}

Auteur correspondant : Savadogo Mamoudou, Email : savadoma@gmail.com

\section{Résumé}

Les sepsis constituent un problème de santé publique dans le monde, particulièrement dans les pays en développement. Cette étude a pour objectif de décrire les caractéristiques épidémiologiques, cliniques, et évolutives des sepsis observés au service des maladies infectieuses du CHU Yalgado Ouédraogo.

Patients et méthode : il s'agit d'une étude transversale descriptive à collecte rétrospective portant sur les patients souffrant de sepsis hospitalisés dans le service des maladies infectieuses du CHU Yalgado Ouédraogo de

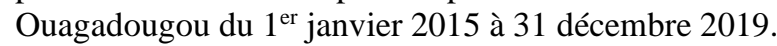

Résultats : Un total de 81 dossiers a été colligé. Les patients provenaient majoritairement de la ville de Ouagadougou (96\%). L'âge moyen était de 32 ans \pm 9 . Quarante-sept patients étaient de sexe masculin contre 34 de sexe féminin soit un sex ratio $=1,4$. Douze pour cent des patients étaient infectés par le VIH. Sur le plan clinique, la fièvre était retrouvée chez $63 \%$ de nos patients. La fréquence cardiaque était supérieure à 90 cycles $/ \mathrm{mn}$ chez $73 \%$ des patients; la fréquence respiratoire était supérieure à 20 cycles $/ \mathrm{mn}$ chez $80 \%$ des patients; une leucopénie $\left(300\right.$ à $\left.3300 / \mathrm{mm}^{3}\right)$ a été retrouvée chez $17 \%$. L'hyperleucocytose $(12000$ à $31480 / \mathrm{mm}^{3}$ ) a été retrouvée chez $10 \%$. Les signes de sévérité du sepsis étaient retrouvés chez $29,6 \%$ des patients. Les signes de gravité étaient dominés par les défaillances des fonctions supérieures (obnubilation ou coma) et les défaillances de la coagulation (thrombopénie inférieure à $100000 / \mathrm{mm}^{3}$ ). Les causes de sepsis étaient dominées par la dengue (42\%), le paludisme (23\%), et les infections bactériennes (14\%). Les bactéries isolées à l'hémoculture étaient dominées par les entérobactéries $(41,6 \%)$ dont la moitié était productrice de Bétalactamase à spectre élargi (BLSE). Une souche productrice de carbapénèmase a été observée parmi les souches d'Escherichia coli. Seize pour cent des souches bactériennes étaient des souches de Staphylococcus aureus dont une souche méticillinorésistante (SARM). Vingt-deux décès ont été enregistrés soit une létalité de $27 \%$.

Conclusion : la dengue, le paludisme et les infections bactériennes étaient les causes les plus fréquentes de sepsis dans le service des maladies infectieuses. La prévalence élevée de souches productrices BLSE, et l'émergence de souche résistantes aux carbapénèmes sont préoccupantes et imposent la prise de mesure visant à améliorer l'hygiène hospitalière et la prescription des antibiotiques. Aussi la gravité des sepsis commande la mise en place d'unités de soins intensifs dans les services de maladies infectieuses pour leur prise en charge.

Mots clés : choc septique, sepsis, sepsis sévère, CHU Yalgado Ouédraogo, Ouagadougou

\begin{abstract}
Sepsis is a public health issue around the world, particularly in developing countries. The aim of this study is to describe the clinical epidemiological and evolutionary characteristics of sepsis observed in the infectious diseases department of the Yalgado Ouedraogo CHU.

Patients and method: this is a descriptive cross-sectional study with retrospective collection with a descriptive aim on sepsis patients hospitalized in the infectious diseases department of the CHU Yalgado Ouédraogo of Ouagadougou from January 1, 2015 to December 31, 2019.

Results: A total of 81 files were collected. Most of the patients came from the town of Ouagadougou (96\%). The mean age was $32 \pm 9$ years. Forty-seven patients were male against 34 female, representing a sex ratio $=1.4$. Twelve percent of the patients were infected with HIV. Clinically, fever was found in $63 \%$ of our patients. Heart rate was greater than 90 cycles / $\min$ in $73 \%$ of patients; the respiratory rate was greater than 20 cycles / $\min$ in $80 \%$ of patients; leukopenia (300 to $\left.3300 / \mathrm{mm}^{3}\right)$ was found in $17 \%$. Hyperleukocytosis $(12,000$ to $31,480 /$ $\mathrm{mm}^{3}$ ) was found in $10 \%$. Signs of the severity of sepsis were found in $29.6 \%$ of patients. Signs of severity were dominated by failure of higher functions (obnubilation or coma) and failure of coagulation (thrombocytopenia less than $\left.100,000 / \mathrm{mm}^{3}\right)$. The causes of sepsis were dominated by dengue (42\%), malaria (23\%), and bacterial infections (14\%). Bacteria isolated in blood culture were dominated by Enterobacteriaceae (41.6\%), half of which produced Extended Spectrum Betalactamase (ESBL). A carbapenemase-producing strain has been observed among strains of Escherichia coli. Sixteen percent of the bacterial strains were strains of
\end{abstract}


Staphylococcus aureus including a methicillin-resistant (MRSA) strain. Twenty-two deaths have been recorded, i.e. a fatality of $27 \%$.

Conclusion: dengue, malaria, and bacterial infections were the most common causes of sepsis in the infectious disease department. The high prevalence of ESBL-producing strains, and the emergence of strains resistant to carbapenems, are worrying and require measures to be taken to improve hospital hygiene and the prescription of antibiotics. Also the severity of sepsis requires the establishment of intensive care units in infectious disease departments for their management.

Key words: sepsis, severe sepsis, septic shock, Yalgado Ouedraogo CHU, Ouagadougou

\section{Introduction}

Le sepsis se définit comme une dysfonction d'organe consécutive à une réponse inappropriée de l'organisme à une infection [1-4]. Il constitue un véritable problème de santé publique dans le monde où un décès sur cinq lui est attribué. C'est l'une des principales causes de décès dans les pays en développement où sa létalité atteint 85\%. En 2017 il a touché 49 millions de personnes et tué 11 millions [5,6] et l'Organisation Mondiale de la Santé (OMS) a fait de sa prévention une priorité [57]. Les enfants de moins de cinq ans et les personnes âgées de plus de 65 ans sont les plus exposés $[8,9]$. Ce sont des urgences médicales dont un diagnostic précoce et un traitement adapté permettent de prévenir l'évolution vers le choc septique qui est pourvoyeur d'une lourde létalité [10-12]. L'objectif de notre étude est de décrire les caractéristiques épidémiologiques, cliniques, et évolutives des cas de sepsis hospitalisés dans le service des maladies infectieuses.

\section{Patients et Méthode}

Il s'est agi d'une étude transversale descriptive à collecte rétrospective portant sur les patients souffrant de sepsis hospitalisés dans le service des maladies infectieuses du CHU Yalgado Ouédraogo de Ouagadougou du $1^{\text {er }}$ janvier 2015 au 31 décembre 2019. Le sepsis a été défini comme toute dysfonction d'organe menaçant le pronostic vital et causée par une réponse inappropriée de l'hôte à une infection. Le choc septique est défini par l'association de trois critères qui sont le sepsis, le besoin de drogues vasopressives pour maintenir une pression artérielle moyenne supérieure à $65 \mathrm{mmHg}$ et des lactates supérieurs à $2 \mathrm{mmol} / \mathrm{l}$ malgré un remplissage adéquat $[10,12]$.

Etaient inclus les patients souffrant de sepsis et disposant d'un bilan biologique de confirmation. Ont été exclus, les patients souffrant de sepsis dont l'étiologie n'a pas pu être précisée.

\section{Résultats}

Un total de 81 dossiers a été colligé. Les patients provenaient majoritairement de la ville de Ouagadougou (96\%). L'âge moyen des patients était de 32 ans \pm 9 . Quarante-sept patients étaient de sexe masculin contre 34 de sexe féminin soit un sex ratio $=1,4$. Douze pour cent de nos patients vivaient avec le VIH. Sur le plan clinique, la fièvre était présente chez $63 \%$ des patients. Elle était supérieure ou égale à $39^{\circ} \mathrm{C}$ chez $54 \%$ d'entre eux. La fréquence cardiaque était supérieure à 90 cycles/mn chez $73 \%$ des patients; la fréquence respiratoire était supérieure à 20 cycles/mn chez $80 \%$ des patients; une leucopénie (300 à 3 $300 / \mathrm{mm}^{3}$ ) a été retrouvée chez 14 patients $(19 \%)$. L'hyperleucocytose (12 000 à $31480 / \mathrm{mm}^{3}$ ) a été retrouvée chez huit patients $(24 \%)$. Les signes de sévérité du sepsis étaient retrouvés chez 24 patients $(29,6 \%)$ : huit cas de défaillance des fonctions supérieures (obnubilation ou coma), sept cas de défaillance de la coagulation (thrombopénie inférieure à $100000 / \mathrm{mm}^{3}$ ), trois cas de défaillance de la fonction rénale (créatinémie supérieure à 177 micromol/l), trois cas de défaillance de la fonction circulatoire (TA $\leq 90 / 40 \mathrm{mmHg}$ ), un cas de défaillance de la fonction respiratoire (fréquence respiratoire supérieure à 50 cycles/mn), un cas de défaillance de la fonction hépatique (hyperbilirubinémie $>34 \mu \mathrm{mol} / \mathrm{l}$ ) et un cas de défaillance hématologique (thrombopénie inférieure à $20000 / \mathrm{mm}^{3}$ ). Les principales étiologies du sepsis étaient représentées par la dengue (42\%), le paludisme grave $(23 \%)$ et les infections bactériennes $(14 \%)$. Les bactéries isolées à l'hémoculture étaient dominées par les entérobactéries $(41,6 \%)$ dont la moitié était productrice de Bétalactamase à spectre élargi (BLSE) avec une souche d'E. coli productrice de carbapénèmase. Seize pour cent des souches isolées étaient représentées par Staphylococcus aureus dont une souche méticillinorésistante (SARM). La figure 1 présente la répartition des bactéries isolées au cours des sepsis. Vingt-deux cas de décès ont été enregistrés soit une létalité de $27 \%$.

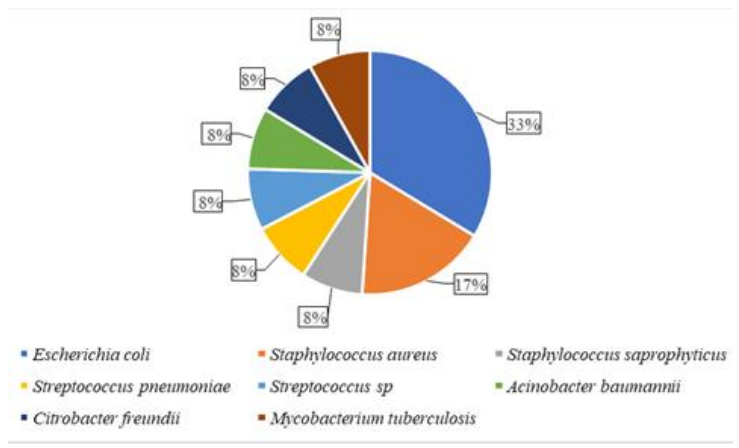

Figure 1 : répartition des bactéries isolées au cours des sepsis 


\section{Discussion}

Les sepsis sont des urgences médicales de diagnostic et de traitement. Ils constituent un problème de santé publique et leur prise en charge grève les budgets des services de santé [1,7]. Dans notre étude le virus de la dengue et l'hématozoaire $\mathrm{du}$ paludisme étaient les étiologies les plus fréquentes de sepsis, contrairement à ce qui est rapporté dans la littérature où sont fréquemment incriminées les bactéries $[9,13,22]$. Nos résultats corroborent les résultats de plusieurs études qui avaient rapporté des cas de sepsis associés à la dengue ou au paludisme [15-18]. La fréquence élevée de la dengue dans notre étude s'explique par les flambées d'épidémie que le Burkina a connues en 2016 et en 2017. L'infection à VIH avec son corollaire d'immunodépression prédispose aux sepsis. En effet $12 \%$ de nos patients étaient infectés par le VIH. Ce taux est en deçà de ce qu'avaient rapporté Ki-Zerbo et Yao respectivement au Burkina Faso et en Côte d'Ivoire [17,18]. Comme dans la série de Seydi au Sénégal, dans notre étude, les entérobactéries dominaient les étiologies bactériennes des sepsis [19]. La majorité des entérobactéries étaient productrice de BLSE avec une souche productrice de carbapénèmase. Selon Vodovar et al., les entérobactéries productrices de bêta-lactamases à spectre élargi (EBLSE), sont aujourd'hui les bactéries multirésistantes (BMR) majoritaires. Elles sont à l'origine d'infections potentiellement sévères $[20,23]$. Les carbapénèmes constituent le traitement de dernier recours au cours de ces infections mais l'émergence d'entérobactéries productrices de carbapénémase est une préoccupation pour les cliniciens. Il en est de même de l'émergence des souches de $S$. aureus résistants à la méticilline (SARM). Ouédraogo au Burkina Faso, expliquait que la résistance aux antibiotiques dans notre contexte était favorisée par les conditions d'hygiène précaires et l'usage abusif et très peu contrôlé des antibiotiques. Ce qui est responsable d'une pression de sélection bactérienne [21,23]. Dans notre étude, les bactéries isolées étaient dominées par Escherichia coli et Staphylococcus aureus. Le même constat a été fait par plusieurs études européennes [22,23]. Les bactériémies à Staphylococcus aureus étaient retrouvées chez $25 \%$ des patients dans notre série. Ce taux est comparable à ce qu'a retrouvé Lagier en France [23] qui expliquait par ailleurs que le taux élevé de bactériémie à Staphylococcus aureus est dû à l'augmentation des procédures invasives en milieu de soins [23]. La lourde létalité due aux sepsis, relevée dans notre étude est aussi constatée par plusieurs études africaines et asiatiques $[18,22,24,25]$. Cette létalité était plus importante chez les patients infectés par le VIH comme relevée par plusieurs auteurs $[8,22]$.
Conclusion : les sepsis sont d'étiologies multiples. $\mathrm{La}$ dengue, le paludisme et les infections bactériennes étaient les plus incriminés. Leur pronostic reste dépendant à la fois de la rapidité du diagnostic et de l'instauration rapide d'un traitement adapté au germe en cause. Leur prise en charge est confrontée à la résistance aux antimicrobiens. Leur prévention passe par l'application des mesures d'hygiène et de prévention contre les infections en milieu de soins, l'amélioration de l'hygiène et de l'assainissement. En cas de sepsis, la rapidité de la mise sous traitement et la prise en compte de la sensibilité aux antimicrobiens, sont des éléments fondamentaux de la stratégie thérapeutique. Il importe donc de mettre en place dans les structures de soins, des unités de soins intensifs pour une bonne prise en charge des cas de sepsis. Devant une hémoculture négative, le clinicien devra penser à des étiologies autres que bactériennes.

\section{Conflit d'intérêt : Aucun}

\section{Références}

1) Silvano E, Simone GD, Boccia G, Caro FD, Pagliano P. Sepsis and septic shock: New definitions, new diagnostic and therapeutic approaches. J Glob Antimicrob Resist. 2017 ; 10 $: 204-12$.

2) Nunnally ME. Sepsis for the anaesthetist. Br J Anaesth. 2016 ;117(3) : 44-51.

3) Minasyan H. Sepsis and septic shock: Pathogenesis and treatment perspectives. J Crit Care. $2017 ; 40$ $: 229-42$

4) Fethi Gül, Mustafa Kemal Arslantas et al. Changing Définitions of Sepsis. Turk J of anaesthesiol and Reanim. 2017 ; 45(3) :129

5) https://www.who.int/news-room/factsheets/detail/sepsis consulté le 05 octobre 2020.

6) Cecconi M, Evans L, Levy M, Rhodes A. Sepsis and septic shock. Lancet. 2018 ;392 (10141) :7587.

7) Seydi M, Soumaré M, Sow AI, Diop BM, Sow PS. Aspects actuels des bactériémies à Salmonella à la clinique des maladies infectieuses Ibrahima Diop Mar du centre hospitalier national de Fann (Senegal). Med et Mal infect. 2004 ; 35(1) :23-7.

8) Rowe TA, Koy JMM. Sepsis in Older Adults. Infect Dis Clin North Am. 2017; 31(4):731-42.

9) Teparrukkul P, Hantrakun V, Imwong $M$, Teerawattanasook N, Wongsuvan G, Day N P,et al. Utility of qSOFA and modified SOFA in severe malaria presenting as sepsis. PLoS One. 2019; 14(10). 
10) Napolitano L M. Sepsis 2018: Definitions and Guideline Changes. Surg Infect. 2018 ;19(2) :117125.

11) Le Conte P, Montassier E, Potel G, Batard E, Le Conte $P$. Prise en charge des états septiques sévères chez l'adulte aux urgences. Ann fr de médecine d'urgence. $2014 ; 4$ (4) :242-248.

12) Kendra M B. Summary of the 2016 International Surviving Sepsis Campaign: A Clinician's Guide. Crit Care Nurs Clin North Am. 2018 ;30(3) :311321

13) Lin GL, McGinley JP, Drysdale SB, Pollard AJ. Epidemiology and Immune Pathogenesis of Viral Sepsis. Front Immunol. 2018; 9:2147.

14) Southeast Asia Infectious Disease Clinical Research Network. Causes and outcomes of sepsis in southeast Asia: a multinational multicentre crosssectional study. Lancet Glob Health.2017 ;5(2) : $157-167$

15) Teparrukkul P, Hantrakun V, Day N, Eoin West T, Direk Limmathurotsakul. Management and outcomes of severe dengue patients presenting with sepsis in a tropical country. PLoS One.2017;12(4).

16) Stije J L, Aniruddha G, Katherine A P, Subash M, Luigi P, Hugh W F K et al. Point-of-care lung ultrasound for the detection of pulmonary manifestations of malaria and sepsis: An observational study. PLoS One. 2018;13(12).

17) Kouamé HY, Tanon A K. Etude comparative de l'insuffisance rénale aiguë communautaire chez le sujet VIH positif et sujet VIH négatif : expérience d'un service de médecine interne à Abidjan (Côte d'Ivoire). Néphrologie et Thérapeutique. 2017 ;13(3), 168-175.

18) Ki-Zerbo G A, Sawadogo A B, Kyelem N, Zoubga A, Thiombiano R, Durand G. Bactériémie à entérobactéries et infection au virus de l'immunodéficience humaine : étude de 26 cas au centre hospitalier national de Bobo-Dioulasso (Burkina Faso). Méd et Mal infect. $2000 ; 30(12): 753-756$.

19) Seydi M, Sow P S, Soumaré M, Ndour C T, Dia $N$ M, DIOP BM, Badiane S. Les bactériémies au cours du sida à Dakar, Sénégal. Méd et Mal infect. 33(6) :323-326.

20) Vodovar D. G, Marcadé L, Raskine I, Malissin B. Mégarbane. Entérobactéries productrices de bêtalactamases à spectre élargi : épidémiologie, facteurs de risque et mesures de prévention. Rev. Med. Interne. 2013;34( 11) : 687-693.

21) Ouédraogo AS. Prévalence, circulation et caractérisation des bactéries multirésistantes au Burkina Faso. Montpellier 2016. Université de Montpellier: thèse de doctorat, Biologie Santé, sous la direction de Godreuil, Sylvain Carrier.

22) CMIT. Bactériémie et fongémie de l'adulte et l'enfant. In E Pilly : Vivactis Plus Ed. 2018 ; 285287.

23) Lagier JC, Letranchant L, Selton-Suty C, Nloga J, Alauzet C, Carteaux J P, May T, DocoLecompte T. Bactériémie et endocardite à Staphylococcus aureus. Ann de cardiol Angéiol. 2008; 57( 2):71-77.

24) Shah S, Kaul A, Jadhav Y , Shiwarkar G. Clinical outcome of severe sepsis and septic shock in critically ill children. Trop Doct. 2020;50(3):186-190.

25) Bone RC, Balk K A, Cerra FB, Dellinger RP. Definition for sepsis and organ failure and guidelines for use of innovative therapies in sepsis. The ACCP/SCCM consensus conférence committee. American college of Chest physicians/society of critical Care Medecine. Chest.1992; $101: 1644-55$. 